\title{
Driftless gas proportional scintillation counter pulse analysis using digital processing techniques ${ }^{\dagger}$
}

\author{
P. C. P. S. Simões, J. M. F. dos Santos* and C. A. N. Conde \\ GIAN-Departamento de Física, Universidade de Coimbra, P-3004-516 Coimbra, Portugal
}

Received 27 July 2000; Accepted 29 May 2001

\begin{abstract}
A driftless gas proportional scintillation counter (GPSC) of simple design, using digital pulse-signature analysis, is described. The digital pulse acquisition and processing techniques are shown to be a simple and effective method to determine pulse time duration and to perform pulse amplitude correction in driftless GPSCs. This technique allows the application of driftless GPSCs to x-ray spectrometry up to $60 \mathrm{keV}$. Although the pulse throughput achieved by the digital pulse-height analyser in this application is limited to about $2 \mathrm{kHz}$, total interaction rates above $20 \mathrm{kHz}$ can be tolerated by the detector without significant degradation of the pulse-height distributions obtained. Copyright $\subset 2001$ John Wiley \& Sons, Ltd.
\end{abstract}

\section{INTRODUCTION}

Gas proportional scintillation counters (GPSCs) have been widely applied in important areas such as x-ray astronomy, medical instrumentation, high-energy physics and energydispersive $x$-ray fluorescence analysis. ${ }^{1-6}$ They combine room temperature operation with large detection areas and high counting rates without space charge effects. ${ }^{1,6}$ The importance of GPSCs has led to continuous research efforts to elucidate their working principles and improve their performance.

The preferred gas medium is a noble gas, usually xenon, chosen for its large photoionization cross-section and high scintillation efficiency. In conventional GPSCs the x-rays interact preferentially in a region with an applied weak electric field, below the threshold for the gas excitation-the drift region. Under the influence of this field, the resulting primary electron cloud drifts towards a well-defined scintillation region where the electric field is high enough for the drifting electrons to excite but not ionize the gas atoms, producing a characteristic vacuum ultraviolet (VUV) scintillation pulse as a result of deexcitation of the gas atoms. Each primary electron gives rise to a very large number of VUV photons, resulting in a large signal amplification, without space charge accumulation. The number of VUV photons is proportional to the number of primary electrons, and hence to the $\mathrm{x}$-ray energy. If the x-rays interact in the drift region, the amount of the resulting scintillation is independent of the position where the $\mathrm{x}$-ray interaction occurs.

*Correspondence to: J. M. F. dos Santos, GIAN-Departamento de Física, Universidade de Coimbra, Rua Larga, P-3004-516 Coimbra, Portugal. E-mail: cesar@gian.fis.uc.pt

${ }^{\dagger}$ Presented at the European Conference on EDXRS, Krakow, Poland, 18-23 June 2000.

Contract/grant sponsor: Fundação para a Ciência e a Tecnologia

(FCT); Contract/grant number: POCTI/43527/99.
However, the interaction of $\mathrm{x}$-rays in a region with a weak electric field can be disadvantageous, in particular for x-ray interactions near the detector radiation window, since some primary electrons may be lost to the window, owing to diffusion. This effect is important for soft $\mathrm{x}$-rays with very low penetration depths in the gas. The pulseheight spectra of a GPSC for such x-ray energies may exhibit distortions represented by a significant departure from the Gaussian shape output in the low-amplitude region owing to a tail that extends down to very low amplitudes. ${ }^{7}$ It was shown that this effect is very sensitive to the electric field in the region near the radiation window and is reduced with increasing electric field.

For these reasons, a driftless GPSC design was developed: ${ }^{8-11}$ a GPSC without a drift region, where the $\mathrm{x}$-rays are directly absorbed in the scintillation region, in contrast to conventional GPSCs where x-rays are preferentially absorbed in a preceding drift region. Owing to the high electric field in the scintillation region, the degrading influence of primary electron loss to the detector radiation window ${ }^{7}$ is reduced when compared with the conventional GPSC operation. Additionally, several other disadvantageous effects such as primary electron loss due to impurities and to the low-voltage grid and the lateral diffusion of the primary electron cloud are also reduced. , $^{12}$ Also, the driftless design is attractive for timing applications where a prompt detector response and/or a steep pulse rise is needed.

However, in a driftless GPSC the observed light amplitude is dependent on the distance covered by the primary electron cloud in the scintillation region, and hence on the depth of the interaction of the incoming x-ray photon. The light pulse time duration is proportional to this distance and allows pulse amplitude normalization, restoring the proportionality between the normalized pulse amplitude and the corresponding x-ray photon energy.

For low-energy x-rays with absorption lengths much smaller than the scintillation region thickness, pulse time 
duration analysis is not necessary, owing to the small effect of the different $x$-ray penetration depths on the distance covered by the primary electron cloud in the scintillation region, and thus on the scintillation light produced. ${ }^{11}$ Nevertheless, if the low-energy x-rays are detected together with high-energy $\mathrm{x}$-rays, the low-energy peaks will be superimposed on a high-level background, which can be efficiently eliminated by using pulse discrimination techniques. Furthermore, the applicability and the advantages of a driftless GPSC design extend to higher energy ranges, ${ }^{9,10}$ where the observed light amplitude must be corrected.

So far, scintillation time duration analysis has been implemented with analogue signal processing requiring sophisticated electronics, which may be a limitation when dealing with fast pulse processing, ${ }^{10}$ a drawback for practical applications. However, when using digital processing techniques, such pulse time duration analysis can be easily implemented using a digital pulse-height analyser (DPHA) with high versatility and without the requirement for additional electronics.

Recent studies investigated the applications of digital pulse processing techniques to $\mathrm{x}$-ray spectrometry with conventional GPSC ${ }^{13-15}$ using a DPHA. The application of these techniques proved to be a simple but effective method for improving the detector performance by background suppression and peak enhancement in x-ray spectrometry applications. X-ray pulse-height distributions were improved on the basis of digital pulse rise-time discrimination ${ }^{13,14}$ or digital pulse time duration discrimination. ${ }^{15}$ These techniques can also be applied with advantage to driftless GPSCs.

In this work, we investigated the application of digital pulse-processing techniques to driftless GPSCs. The pulse time duration was assessed to determine the x-ray penetration depth and perform pulse-amplitude correction. The x-ray energy range of application and the performance obtained with this technique for energy-dispersive $x$-ray spectrometry (EDXRS) were evaluated.

\section{EXPERIMENTAL}

\section{Detector}

The driftless GPSC is shown in Fig. 1. It has a $10 \mathrm{~cm}$ diameter $\times 2.5 \mathrm{~cm}$ deep scintillation region and is filled with xenon at $10^{5} \mathrm{~Pa}$, which is continuously purified through getters
(SAES St707 type) at $150^{\circ} \mathrm{C}$, and maintained in circulation by convection. The upper part of the detector body is made of Macor, intended to insulate the detector radiation window and holder, which are biased at negative high voltage. The window holder is made of stainless steel and is epoxied to the Macor and to a $7.5 \mu \mathrm{m}$ thick, $8 \mathrm{~mm}$ diameter Kapton window aluminized on the inner side to ensure electrical conductivity. The lower part of the detector body is made of stainless steel and is connected to the gas circulation tubing. The bottom of the detector body is a Macor disc epoxied to the PMT enclosure and to the detector wall.

The scintillation region is delimited by the detector radiation window holder and the photomultiplier (PMT) window. The window holder and the respective Macor piece were designed to ensure a uniform electric field in this region. The PMT is a $5.1 \mathrm{~cm}$ diameter linear focused EMI D676QB (an eight-dynode version of the EMI 9266QB PMT). Using vacuum deposition techniques, a chromium grid (about $100 \mu \mathrm{m}$ linewidth and $1000 \mu \mathrm{m}$ spacing) and a continuous chromium film were deposited on the PMT window and on the lateral surface, respectively. These chromium films are connected to the PMT photocathode pin in order to inhibit any voltage difference between the photocathode and its window external surface. This design enables the PMT to be in direct contact with the scintillation region, eliminating the need for an extra optical window and maximizing the light collected by the PMT.

A $10 \mathrm{kV}$ voltage difference was applied to the scintillation region corresponding to a reduced electric field (the electric field divided by the gas pressure) of about $3.8 \mathrm{~V} \mathrm{~m}^{-1} \mathrm{~Pa}^{-1}$. This value, although adequate, might be increased by about $20-30 \%$ for optimum performance conditions, ${ }^{16}$ resulting in a similar increase in the amount of the scintillation light produced in the detector. However, we were not able to achieve the higher reduced fields because of the unavailability of a larger high-voltage power supply.

\section{Digital signal processing electronics}

The detector pulses were fed through a charge preamplifier (Canberra 2005), with $4.5 \mathrm{mV} \mathrm{pC}^{-1}$ sensitivity, to an amplifier (HP5582A) operated with a very short shaping time (50 ns) and to the DPHA board where they were digitized and further processed.

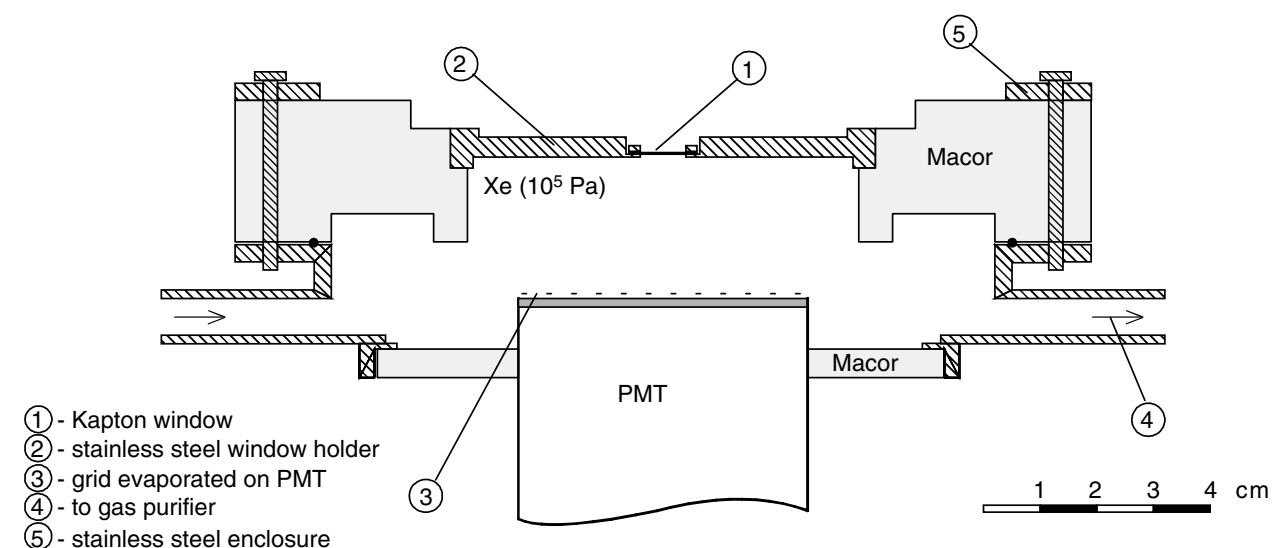

Figure 1. Schematic diagram of the driftless GPSC used in this work. 
The shaping times (50 ns integration and differentiation) were selected to maximize the signal-to-noise ratio and to obtain an electric pulse that closely resembled the scintillation light burst. The pulse total area, the relevant parameter for x-ray analysis, was calculated numerically after a fast digitization.

The DPHA used in this work was composed by a commercially available digitizer PCI board (SignatecPDA12A $)^{17}$ and the host computer where all the data processing was performed.

The digitizer board has a 12 bit ADC whose sampling rate is software selectable up to the maximum of $125 \mathrm{MHz}$. It has complex triggering capabilities including data acquisition after or before a certain time interval measured from the instant when the voltage trigger level is reached. The input voltage range is $0-1 \mathrm{~V}$ but only $90 \%$ of the ADC fullscale is used as a d.c. offset is added to the signal prior to the digitization. The trigger voltage level is adjusted to be just above the electric noise level $(\sim 20 \mathrm{mV})$ and the pulse digitization begins $3 \mu$ s before the trigger level is reached. A sampling frequency of $20 \mathrm{MHz}$ is used, thus 20 points are sampled for a $1 \mu$ s pulse duration. This sampling frequency is a good compromise between the performance obtained and the DPHA pulse-processing time, leading to a maximum throughput of 2000 counts per second.

In a typical run, each pulse is processed by a series of algorithms as follows. A three-point median filter ${ }^{18}$ and a moving average filter ${ }^{18}$ smooth the digitized pulse samples, reducing high-frequency oscillations. These filters are required so that the pulse duration value calculated by the
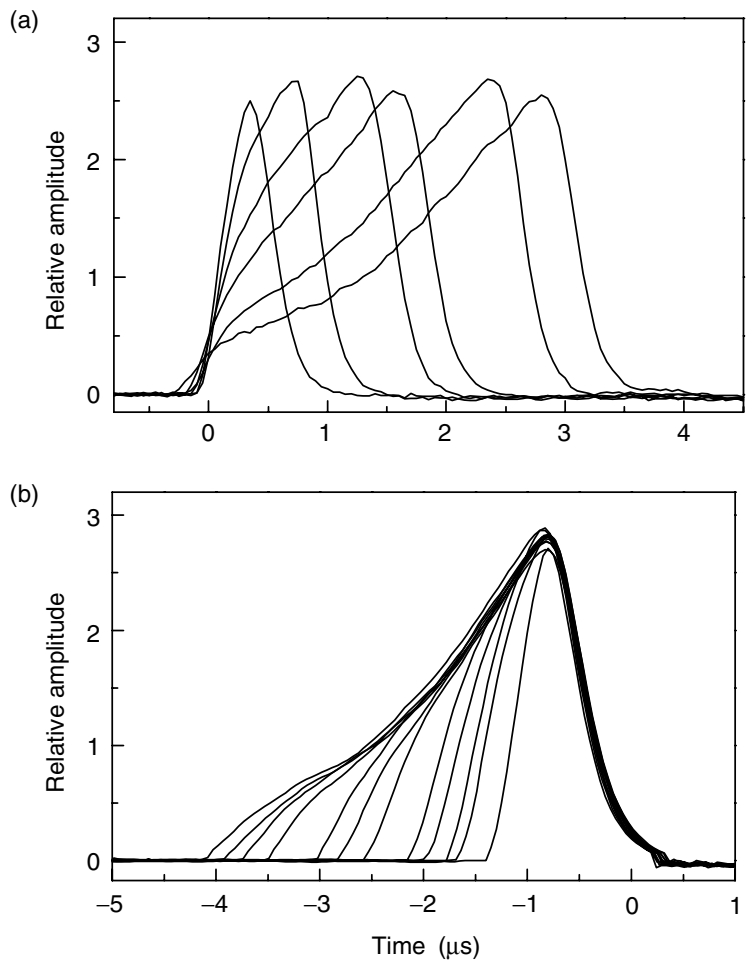

Figure 2. Typical pulse shapes (a) before and (b) after applying the filtering algorithms. The selected pulses correspond to $22.1 \mathrm{keV} \mathrm{x}$-ray interactions at different depths. The filtered pulses were aligned by the leading edge. algorithm will not be affected by the spurious high-frequency ringing originating from the electronic components. Figure 2 shows typical pulse shapes before and after applying the filtering algorithms. The selected pulses correspond to $22.1 \mathrm{keV}$ x-ray interactions at different depths. The filtered pulses are aligned by the leading edge. This edge presents the same shape for every pulse since all the primary electron clouds have about the same size upon reaching the photomultiplier tube. The pulse amplitude is taken as the total area of all the pulse samples whose value is just above the noise level. The pulse time duration is the pulse duration obtained after cutting off the $10 \%$ pulse initial and final areas. A pulse is rejected whenever any sampled value overshoots the ADC scale.

Pulse discrimination is performed on the basis of the pulse duration. As previously, ${ }^{13-15}$ partial pulse-height distributions discriminated according to different pulseduration are recorded simultaneously.

\section{RESULTS AND DISCUSSION}

Using the above-described electronic system, the detector pulse distributions are decomposed into several partial distributions, obtained according to different pulse time durations. Figure 3 presents the total pulse-height distributions and several partial pulse-height distributions obtained for (a) $5.9 \mathrm{keV}$ x-rays and (b) ${ }^{109} \mathrm{Cd}$ source x-rays. The partial pulse-height distributions, selected using time-window widths of $0.04 \mu \mathrm{s}$, were obtained by counting only pulses with a time duration between 1.0 and $2.1 \mu \mathrm{s}$.
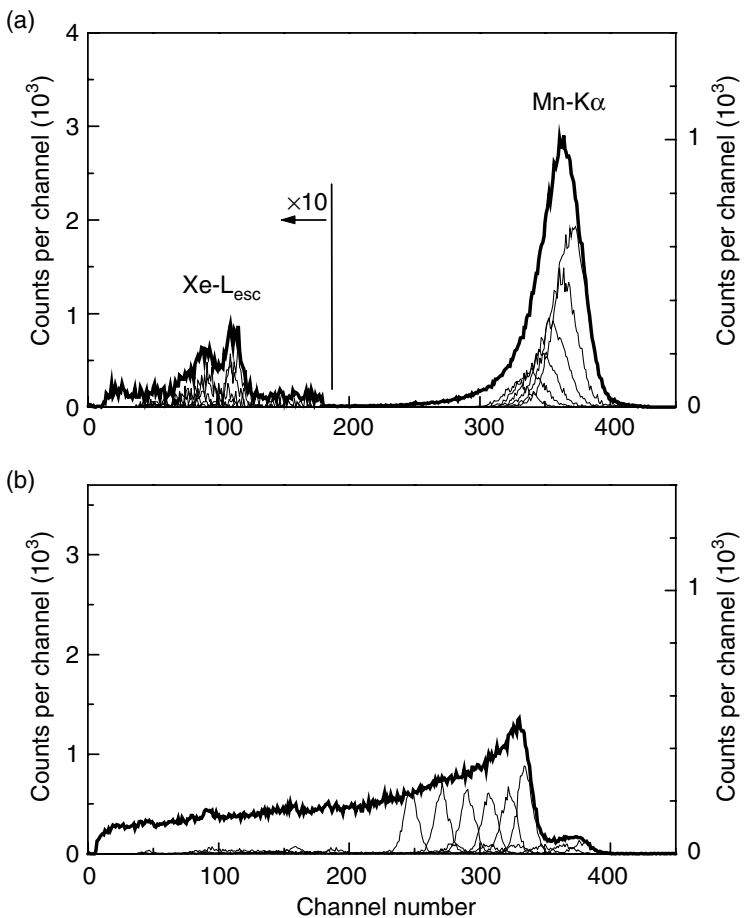

Figure 3. Total pulse-height distributions (thick lines) and several partial pulse height distributions (thin lines) obtained for (a) $5.9 \mathrm{keV} x$-rays and (b) ${ }^{109} \mathrm{Cd}$ source $\mathrm{x}$-rays. The partial pulse-height distributions, selected using time window widths of $0.04 \mu \mathrm{s}$, were obtained by counting only pulses with a time duration between 1.0 and $2.1 \mu \mathrm{s}$. 
(a)

a) Absorption depth $z(\mathrm{~cm})$

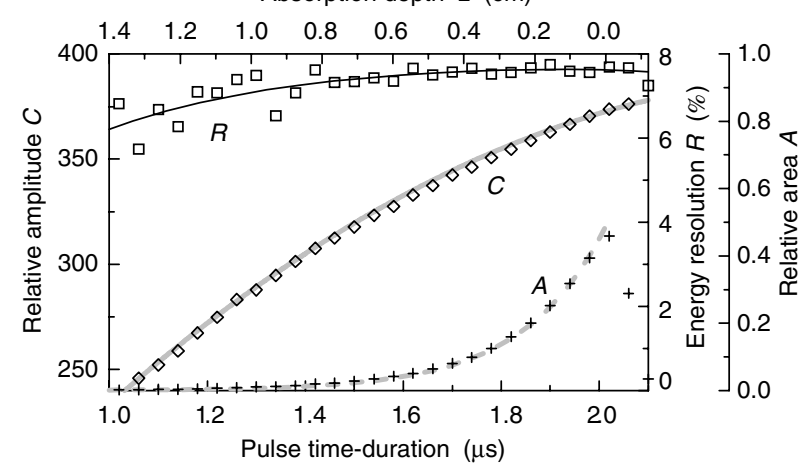

(b)

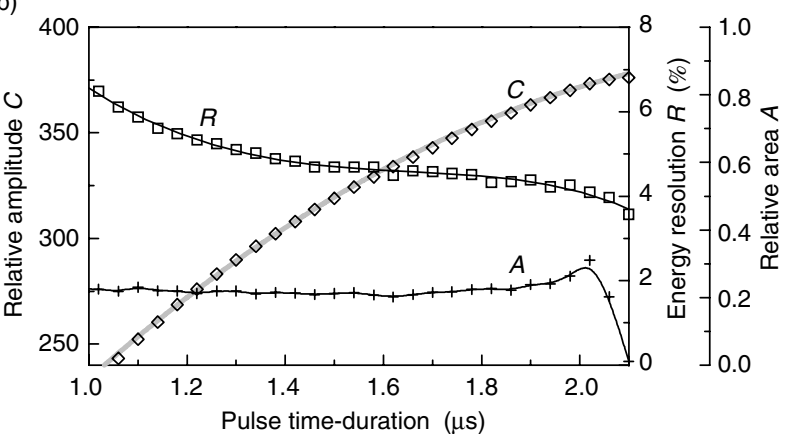

Figure 4. Peak centroid $C$, energy resolution $R$ and peak area $A$ as a function of the selected pulse time duration window for (a) 5.9 and (b) $22.1 \mathrm{keV}$ x-rays. The quadratic function (solid lines) used for amplitude correction is plotted in both (a) and (b). In (a) an exponential fitting $\mathrm{e}^{-z / \lambda}$ (dashed line) to the peak area as a function of the interaction depth $z$ is also depicted.

Figure 4 presents the respective peak centroid $C$, energy resolution $R$ and peak area $A$ as a function of the selected pulse time duration window. The pulse amplitude decreases with decreasing pulse time duration. A good correlation between the pulse amplitude and the pulse time duration is found and a quadratic fitting to these data is used as the amplitude normalization function.

Although the amount of scintillation light produced by the drifting electron cloud is proportional to the drift distance covered by the cloud, and hence to the pulse time duration, the solid angle subtended by the PMT increases for paths nearer the PMT and the collected scintillation light does not hold that proportionality. Instead, this dependence is approximately quadratic, as displayed in Fig. 4 . No dependence of this function on the x-ray energy was found. The average dimension of the primary electron cloud for the different $x$-ray energies is in any case too small, when compared to the drift distances, to have a significant influence on the pulse time duration.

In Fig. 5 the total and partial pulse-height distributions after the amplitude correction has been implemented are depicted. Energy resolutions of 7.8 and $4.5 \%$ for 5.9 and $22.1 \mathrm{keV}$, respectively, were obtained, a performance similar to that of a conventional GPSC.

The different trends of the peak area with the pulse time duration for the different $x$-ray energies (Fig. 4) can be explained in terms of the $\mathrm{x}$-ray absorption length: the peak area dependence on the $\mathrm{x}$-ray penetration depth reflects the
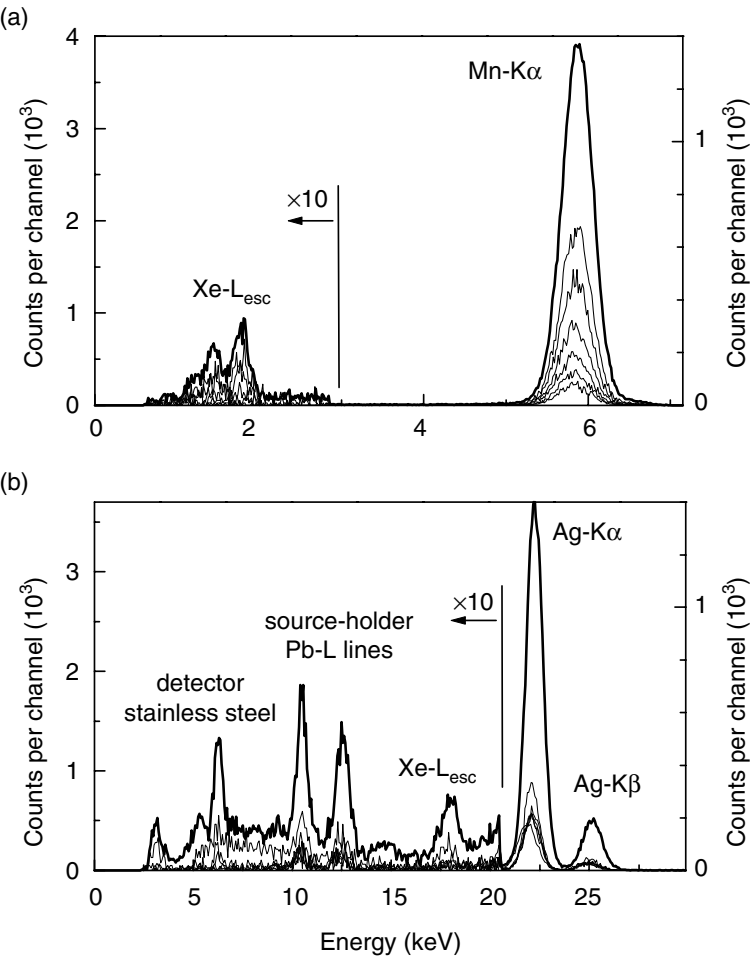

Figure 5. Total (thick lines) and partial (thin lines) pulse-height distributions after amplitude correction obtained for (a) $5.9 \mathrm{keV}$ $\mathrm{x}$-rays and (b) ${ }^{109} \mathrm{Cd}$ source $\mathrm{x}$-rays.

exponential attenuation characteristic of the x-ray beams. For $x$-rays with absorption lengths shorter than the scintillation region depth, an exponential decrease in the peak area with decreasing pulse time duration window is expected, the exponential parameter being equal to the mean absorption depth. Fitting the data of Fig. 4(a) to an exponential function, one obtains a mean absorption depth $\lambda$ of about $2.3 \mathrm{~mm}$ for $5.9 \mathrm{keV}$ x-rays. A mean absorption depth of $0.53 \mathrm{~mm}$ was found for Si K x-rays. These values are in good agreement with the values 2.7 and $0.62 \mathrm{~mm}$, respectively, reported in Ref. 19. For x-rays with absorption lengths much longer than the scintillation region depth, an approximate constant peakarea dependence is expected, as shown in Fig. 4(b). The same trend is observed for the $60 \mathrm{keV} x$-rays.

For high-energy $x$-rays, the energy resolution obtained for the partial spectra increases with decreasing pulse time duration [Fig. 4(b)]. As the distance covered by the primary electron cloud is shorter, the solid-angle fluctuations and UV photon counting statistics become important. This is due to the finite size of the primary electron cloud and to the smaller amount of scintillation light produced ${ }^{20}$ respectively.

To improve the energy resolution achieved, $x$-ray pulses with short time duration, i.e. $x$-rays absorbed in the lower part of the scintillation region, may be rejected, but this results in a decrease in the detection efficiency. Hence a compromise must be established between energy resolution and detection efficiency. Table 1 summarizes the energy resolution, detection efficiency and the corresponding useful scintillation region depth for different pulse time duration rejection thresholds for 10.5 and $22.1 \mathrm{keV}$ x-ray peaks, $\mathrm{Pb} \mathrm{L} \alpha^{1}$ and $\operatorname{Ag~} \mathrm{K} \alpha$, respectively. This compromise can be improved 
Table 1. Energy resolution $\Delta E / E$, detection efficiency and the corresponding useful scintillation region depth for different pulse time duration rejection thresholds for 10.5 and $22.1 \mathrm{keV} x$-ray peaks

\begin{tabular}{|c|c|c|c|c|c|}
\hline \multirow{2}{*}{$\begin{array}{l}\text { Time duration threshold } \\
\qquad(\mu \mathrm{s})\end{array}$} & \multirow{2}{*}{$\begin{array}{l}\text { Xenon thickness } \\
\text { (mm) }\end{array}$} & \multicolumn{2}{|c|}{$\Delta E / E(\%)$} & \multicolumn{2}{|c|}{ Detection efficiency (\%) } \\
\hline & & $10 \mathrm{keV}$ & $22 \mathrm{keV}$ & $10 \mathrm{keV}$ & $22 \mathrm{keV}$ \\
\hline $0.6-2.0$ & 17 & 6.9 & 6.0 & 75 & 17 \\
\hline $1.0-2.0$ & 13 & 6.5 & 5.5 & 65 & 13 \\
\hline $1.2-2.0$ & 10 & 6.2 & 4.8 & 57 & 11 \\
\hline $1.4-2.0$ & 8 & 6.1 & 4.5 & 48 & 8 \\
\hline $1.8-2.0$ & 3 & 6.1 & 4.4 & 23 & 4 \\
\hline $1.9-2.0$ & 1 & 5.9 & 4.3 & 10 & 1 \\
\hline
\end{tabular}

by maximizing the reduced electric field, using a larger diameter PMT or increasing the scintillation region depth.

\section{X-ray spectrometry applications}

Figure 6 depicts the peak centroid and the full width at halfmaximum values of the measured pulse-height distributions obtained by irradiating the detector either with direct radiation from $x$-ray radioactive sources or with fluorescence radiation from single- or multi-element target samples. As can be seen, the good energy linearity of GPSCs still holds after the implementation of pulse amplitude correction. The depicted energy resolution is obtained for a pulse time duration threshold of $1.4 \mu \mathrm{s}$, which corresponds to an x-ray absorption efficiency of a $\sim 1 \mathrm{~cm}$ xenon thick layer.

The deviation from a strict $E^{-1 / 2}$ dependence of the energy resolution is explained by geometric effects that reduce the light collection efficiency or introduce fluctuations in pulse amplitude, e.g. higher average $\mathrm{x}$-ray penetration depth [as discussed regarding Fig. 4(b)] and solid angle effects. The experimental data follow approximately the $E^{-1 / 2}$ trend as shown in Fig. 6.

The detector was irradiated with a ${ }^{55} \mathrm{Fe} x$-ray source, producing different interaction rates between 100 and 20000 counts per second. The energy resolution degraded from 7.7 to 8.4 and to $9.6 \%$ for total count rates of about 400 , 9000 and 20000 counts per second, respectively, while the peak-to-background ratio remained constant.

The pulse-height distributions obtained for the ${ }^{244} \mathrm{Cm}$ and ${ }^{241} \mathrm{Am} x$-ray sources are presented in Fig. 7(a) and (b), respectively. The $\mathrm{Pt}$ L-lines of the ${ }^{244} \mathrm{Cm}$ source result from

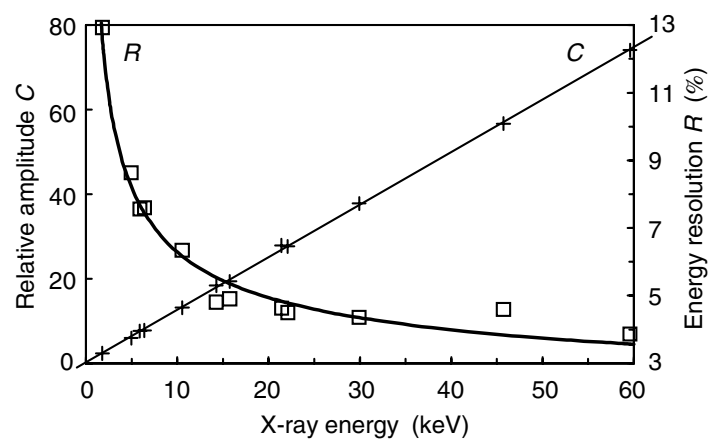

Figure 6. Peak centroid $C$ and full width at half-maximum $R$ values for various $x$-ray energies. Linear fitting (thin line) to the peak-centroid and $E^{1 / 2}$ fitting (thick line) to the calculated energy resolution values.
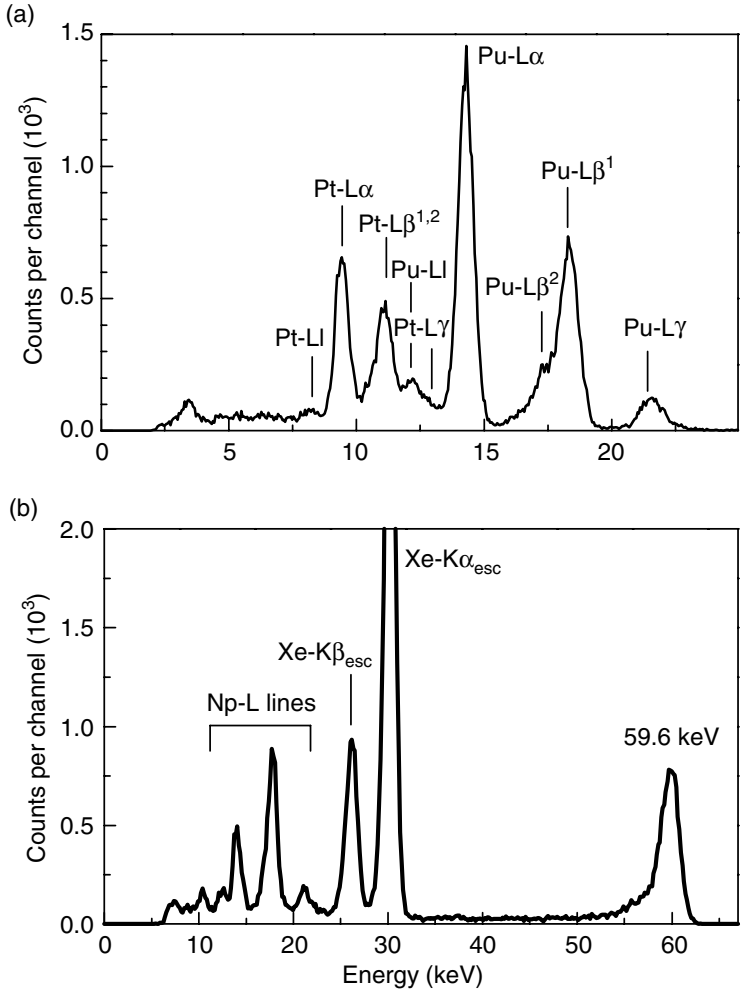

Figure 7. Pulse-height distributions obtained for (a) ${ }^{244} \mathrm{Cm}$ and (b) ${ }^{241} \mathrm{Am} \mathrm{x}$-ray sources after pulse amplitude correction has been applied.

$\mathrm{x}$-ray fluorescence emission of the source holder. The spectral features of the ${ }^{241} \mathrm{Am}$ source include the full-energy peak at $59.6 \mathrm{keV}$, the intense $\mathrm{K} \alpha$ and $\mathrm{K} \beta$ xenon fluorescence escape peaks and the $\mathrm{Np} \mathrm{L}$ x-rays.

X-ray fluorescence spectra were measured for thick samples of raw $\mathrm{Si}$ excited with a ${ }^{55} \mathrm{Fe}$ x-ray source, of a SAES St707 getter $(70 \% \mathrm{Zr}, 5.4 \% \mathrm{Fe}$ and $24.6 \% \mathrm{~V})$ and of dysprosium oxide. The resulting pulse-height distributions are depicted in Fig. 8. Energy resolutions of 13, 7.6, 4.9 and $4.6 \%$ are obtained for the $\mathrm{Si}, \mathrm{Fe}, \mathrm{Zr}$ and Dy. For comparison, the pulse-height distributions obtained with a conventional GPSC are also depicted in Fig. 8. The advantage of a driftless GPSC for soft $x$-rays is evidenced by the undistorted Gaussian shapes of the peaks, with improved energy resolution, and reduced electronic noise tail in the low energy limit when compared with the pulse-height spectra obtained with a conventional GPSC. For higher 
(a)

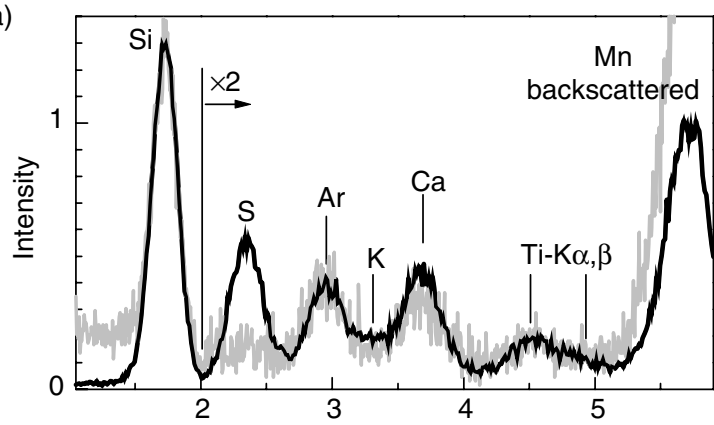

(b)

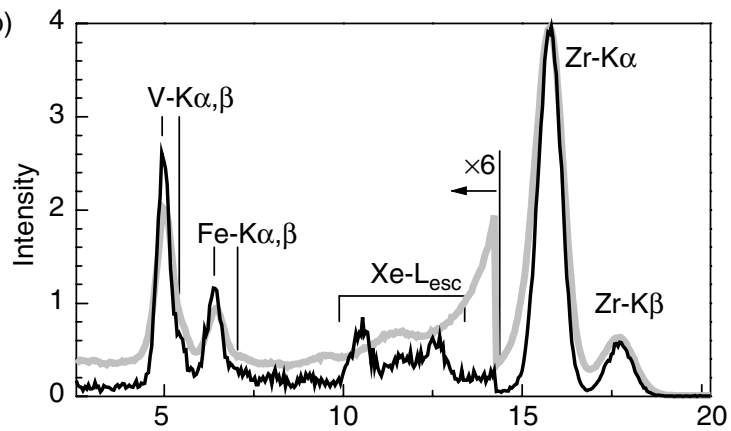

(c)

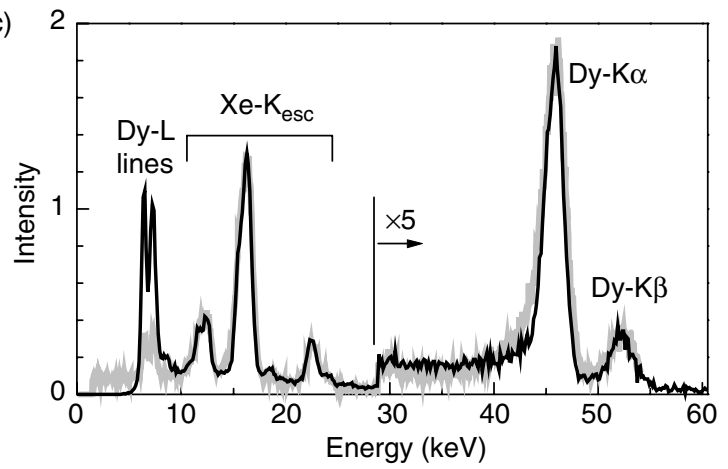

Figure 8. X-ray pulse-height distributions measured for (a) thick samples of raw $\mathrm{Si}$ excited with a ${ }^{55} \mathrm{Fe} x$-ray source, (b) an SAES St707 getter and (c) dysprosium oxide, obtained with the driftless GPSC (black lines) and with a conventional GPSC (grey lines).

energy x-rays, the spectra are similar to those that would be obtained with a conventional, pure xenon GPSC with the advantage of having slightly reduced backgrounds.

\section{CONCLUSIONS}

A GPSC of simple design in a driftless configuration has been presented. The detector performance has been demonstrated to be superior to that of conventional GPSCs with separated drift and scintillation regions, especially for soft x-ray spectrometry. Detector energy resolutions of 13, 7.8 and $4.5 \%$ were obtained for $1.75,5.9$ and $22.1 \mathrm{keV}$ x-rays, respectively. However, the detection efficiency of a driftless GPSC is limited when compared with that which could be achieved with a conventional GPSC, owing to the high voltage requirements for biasing a thick scintillation region, with high electric field, compared with the low electric field demands of a drift region.

Digital pulse acquisition and processing techniques have shown to be a simple and effective method to determine pulse time duration and perform pulse amplitude correction in driftless GPSCs in x-ray spectrometry applications. Additionally, this technique allows efficient pulse time duration discrimination for background reduction of pulse-height distributions. It was demonstrated that this technique allows the application of driftless GPSCs to x-ray spectrometry up to energies above $60 \mathrm{keV}$. The maximum pulse throughput achieved by the DPHA in this application is limited to about 2000 counts per second. However, total interaction rates above 20000 counts per second can be tolerated in the detector without significant degradation of the pulse-height distribution obtained, whereas for conventional pulse formatting techniques the longer shaping times used do not tolerate such high count rates without distortions to the pulse-height distributions obtained.

\section{Acknowledgements}

This work was carried out in the Grupo de Instrumentação Atómica e Nuclear (GIAN) of the Centro de Instrumentação (Unit 217/94) of the Departamento de Física, Universidade de Coimbra. It was supported by the Fundação para a Ciência e a Tecnologia (FCT) through POCTI/43527/99. P. C. P. S. Simões was partially supported by FCT through grant PRAXIS XXI BD/19604/99.

\section{REFERENCES}

1. Policarpo AJPL. Space Sci. Instrum. 1977; 3: 77.

2. Perez-Mendes LBLV. In Instrumentation in Elementary Particle Physics, Fabjian CW, Pilcher JE (eds). World Scientific: Singapore, $1987 ; 149$.

3. Lamb P,Manzo G, Re S, Boella G, Villa G, Andresen R, Sims MR, Klark GF. Astrophys. Space Sci. 1987; 136: 369.

4. Smith A, Bavdaz M. Rev. Sci. Instrum. 1992; 63: 683.

5. Goganov DA, Shultz AA. Nucl. Instrum. Methods A 1994; 394: 151.

6. Varvaritsa, Vikulov IV, Ivashov VV, Panov MA, Filatov VI, Schekin KI. Instrum. Exp. Tech. 1992; 35: 745.

7. Santos FP, dos Santos JMF, Dias THVT, Conde CAN. IEEE Trans. Nucl. Sci. 1995; 42: 611.

8. Simons DJ, de Korte PAJ, Peacock A, Bleeker JAM. Proc. SPIE 1985; 597: 190.

9. Smith A, Peacock A, Kowalski TZ. IEEE Trans. Nucl. Sci. 1987; 34: 57.

10. Simons DG, de Korte PAJ. Nucl. Instrum. Methods A 1989; 277: 642.

11. Barbera M, Collura A, Favata F, Inzerillo G, Mirabello F. Proc. SPIE 1994; 2280: 154.

12. Peacock A, Andresen RD, Leimann EA, Long AE, Manzo G, Taylor BG. Nucl. Instrum. Methods 1980; 169: 613.

13. Simões PCPS, Veloso JFCA, dos Santos JMF, Conde CAN. IEEE Trans. Nucl. Sci. 1997; 44: 521.

14. Simões PCPS, dos Santos JMF, Conde CAN. X-Ray Spectrom. 1997; 26: 182.

15. Simões PCPS, dos Santos JMF, Conde CAN. Nucl. Instrum. Methods A 1999; 422: 341.

16. Borges FIGM, dos Santos JMF, Dias THVT, Santos FP, Rachinhas PJBM, Conde CAN. Nucl. Instrum. Methods A 1999; 422: 321.

17. PDA12A-125 Mhz Waveform Digitizer. Signatec: Corona, CA, 1998; http:/ / www.signatec.com.

18. Press WH, Flannery BP, Tenkolsky SA, Vetterling WT. Numerical Recipes in Pascal. Cambridge: Cambridge University Press 1989; 545: 546; http://www.nr.com.

19. Henke BL, Gullikson EM, Davis JC. At. Data Nucl. Data Tables 1993; 54: 181. http:/ / www-cxro.lbl.gov/optical_constants.

20. Veloso JFCA, dos Santos JMF, Conde CAN. Nucl. Instrum. Methods A 2001; 457: 253. 